\title{
Fourier Transforms in Digital Signal Processing
}

\section{Bai Kang}

\author{
School of Control and Computer Engineering, North China Electric Power University \\ Baoding, 071003, Hebei, China \\ pisa_kang@126.com
}

Keywords: Digital signal processing, Fourier transform, Inherent relationship, Learning effect

\begin{abstract}
Digital signal processing is one of the core courses for several engineering specialties, and its theories include five kinds of Fourier transforms, i.e. FT, FS, DTFT, DFS and DFT. The understanding of the inherent relationship between them will directly affect the learning effect as well as the following courses. In this paper, a new way of Fourier transforms teaching is discussed, i.e. based on FT, the influence on the time(frequency) domain signal when sampling it in frequency(time) domain is analysed, and in this way the relationship between the five transforms is discussed. We hope that a better classroom effect will be achieved.
\end{abstract}

\section{Introduction}

Nowadays, digital signal processing has become a foundation required course for several engineering specialties in the domestic colleges and universities because of the rapid innovation of testing and processing technology based on microprocessors and digital signal processors ${ }^{[1-3]}$.

The core theories of this course include five kinds of Fourier transforms, i.e. Fourier transform(FT), Fourier series(FS), discrete time Fourier transform(DTFT), discrete Fourier series(DFS) and discrete Fourier transform(DFT ${ }^{[4,5]}$. Stack mathematical theories and complex formula derivations increase the students' difficulties in understanding. In addition, most teachers' teaching method of directly giving the conversion formulas but ignoring the inherent relationship between them also increases the burden of students ${ }^{[2,3]}$. Therefore, it is necessary to find a teaching idea to help the students to lay the foundation for the learning contents.

From the structure of the linear space, the literature [1] discusses the physical relationship between DFS and FT by using the concepts of inner product, orthogonal basis and function space structure. Differently, in this paper, based on FT, what happened to a signal in time (frequency) domain when it is sampled in frequency (time) domain is analysed, and then, the relationship between the five Fourier transforms is discussed.

\section{Fourier Transform (FT)}

Let $x(t)$ be a continuous- time signal which belongs to $L_{2}$ space and satisfies Dirichlet conditions, i.e.

$$
\int_{-\infty}^{\infty}|x(t)|^{2} d t<\infty
$$

Then the Fourier transform of $x(t)$ is existed and can be written as below

$$
X(j \Omega)=F(x(t))=\int_{-\infty}^{\infty} x(t) e^{-j \Omega t} d t .
$$

Inverse Fourier transform is defined as

$$
x(t)=F^{-1}(X(j \Omega))=\frac{1}{2 \pi} \int_{-\infty}^{\infty} X(j \Omega) e^{j \Omega t} d \Omega,
$$


where $\Omega=2 f$ is the analog angular frequency in radians / $\mathrm{s}, \mathrm{X}(j \Omega)$ is a function of $\Omega$, known as the spectral density function of $x(t)$.

By definition, the $x(t)$ has finite energy in the time domain. Also, the energy of frequency signal $X(j \Omega)$ is limited since Parseval Theory. Generally, Periodic signal is not a limited energy signal, so the signals we obtained in two domains are both continuous and aperiodic, as shown in Fig.1(a).
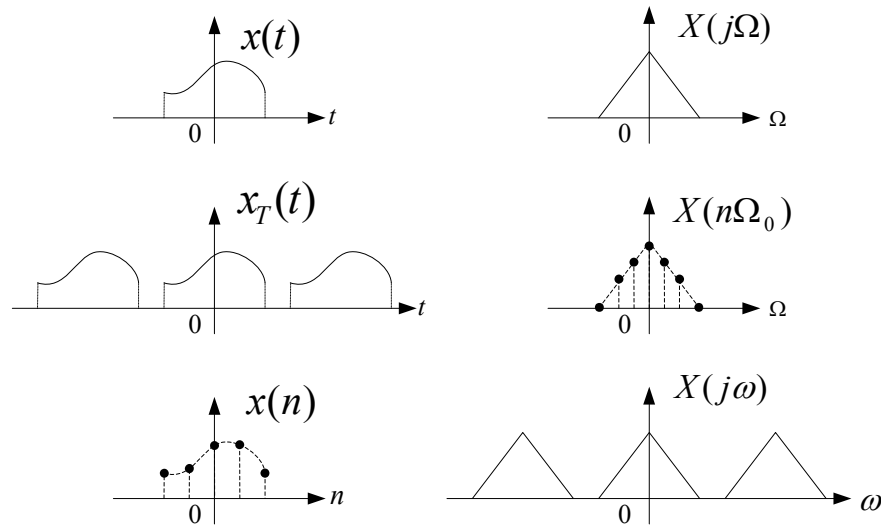

(c) DTFT
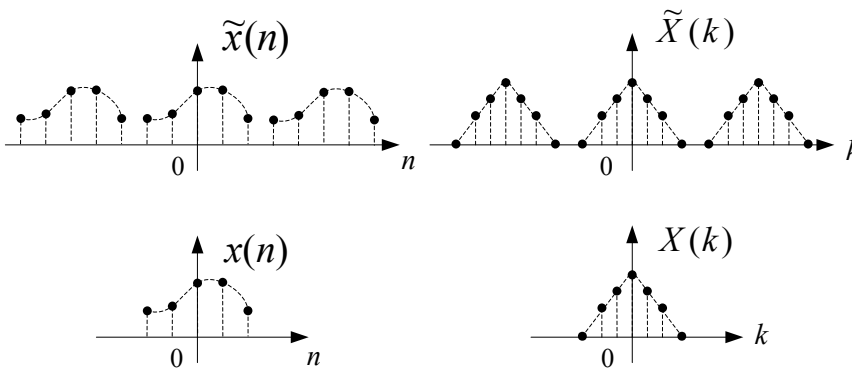

Fig. 1. Illustrations of five Fourier transforms (d) DFS (e) DFT

(a) FT

(b) FS DFS

\section{Sampling in Frequency(Time) Domain Only}

As shown in Fig.1, $x_{T}(t)$ is a time domain periodic signal with period $\mathrm{T}$ and $x(t)$ is one period of $x_{T}(t)$ without the case of aliasing. We let $\delta_{T}(t)$ be the periodic impulse train as

$$
\delta_{T}(t)=\sum_{n=-\infty}^{\infty} \delta(t-n T)
$$

where $\delta(t)$ is the unit impulse function, or Dirac delta function. Then, the Fourier Transform of $\delta_{T}(t)$ is given by

$$
F\left(\delta_{T}(t)\right)=\int_{-\infty}^{\infty} \delta_{T}(t) e^{-j \Omega t} d t=\Omega_{0} \sum_{n=-\infty}^{\infty} \delta\left(\Omega-n \Omega_{0}\right)=\Omega_{0} \delta_{\Omega_{0}}(\Omega),
$$

where $\Omega_{0}=2 \pi / T$. Eq.5 indicates that the Fourier Transform of $\delta_{T}(t)$ is also a periodic impulse string. Since

$$
x_{T}(t)=x(t) * \delta_{T}(t)
$$

we apply the Fourier transform to Eq. 6 and get the following formula

$$
\begin{aligned}
X_{T}(j \Omega) & =F\left(x_{T}(t)\right)=F\left(\left(x(t) * \delta_{T}(t)\right)=X(j \Omega) \cdot \Omega_{0} \delta_{\Omega_{0}}(\Omega)\right. \\
& =\Omega_{0} \sum_{n=-\infty}^{\infty} X(j \Omega) \cdot \delta\left(\Omega-n \Omega_{0}\right)=\Omega_{0} \sum_{n=-\infty}^{\infty} X\left(j n \Omega_{0}\right) \cdot \delta\left(\Omega-n \Omega_{0}\right) .
\end{aligned}
$$


Eq.7 shows that the Fourier transform of the periodic function in time domain is a weighted impulse function string in frequency domain. Let $X\left(n \Omega_{0}\right)=X\left(j n \Omega_{0}\right) / T$, then

$$
X\left(n \Omega_{0}\right)=\frac{1}{T} \int_{-\frac{T}{2}}^{\frac{T}{2}} x(t) e^{-j n \Omega_{0} t} d t=\frac{1}{T} \int_{-\frac{T}{2}}^{\frac{T}{2}} x_{T}(t) e^{-j n \Omega_{0} t} d t .
$$

From the principle of Fourier transform and Eq.7, it follows that

$$
x_{T}(t)=\frac{1}{2 \pi} \int_{-\infty}^{\infty} X(j \Omega) e^{j \Omega t} d \Omega=\frac{1}{2 \pi} \int_{-\infty}^{\infty} \Omega_{0} \sum_{n=-\infty}^{\infty} X\left(j n \Omega_{0}\right) \cdot \delta\left(\Omega-n \Omega_{0}\right) e^{j \Omega t} d \Omega .
$$

Interchanging the order of summation and integration, Eq. 9 can be expressed as

$$
\begin{aligned}
& x_{T}(t)=\frac{1}{2 \pi} \int_{-\infty}^{\infty} \Omega_{0} X\left(j n \Omega_{0}\right) \sum_{n=-\infty}^{\infty} \delta\left(\Omega-n \Omega_{0}\right) e^{j \Omega t} d \Omega \\
& =\frac{1}{2 \pi} \Omega_{0} \sum_{n=-\infty}^{\infty} X\left(j n \Omega_{0}\right) \int_{-\infty}^{\infty} \delta\left(\Omega-n \Omega_{0}\right) e^{j \Omega t} d \Omega \\
& =\sum_{n=-\infty}^{\infty} X\left(n \Omega_{0}\right) e^{j n \Omega_{0} t} .
\end{aligned}
$$

Eq. 8 and Eq. 10 are called the transform pair of FS, while the former is positive transform and the later inverse one. Thus, we can interpret FS this way: sampling frequency signal $X(j \Omega)$ with sampling frequency $\Omega_{0}=2 \pi / T$ leads to the periodic extension of $x(t)$ in the time domain, i.e. $x_{T}(t)$, shown as Fig.1(b).

Similarly, $X_{\Omega_{0}}(j \Omega)$ is a periodic signal in the frequency domain resulting from the convolution of the periodic signal $X(j \Omega)$ with the unit impulse function string $\delta_{\Omega 0}(\Omega)$, i.e.

$$
X_{\Omega_{0}}(j \Omega)=X(j \Omega) * \delta_{\Omega_{0}}(\Omega) .
$$

Convolution property of the Fourier transform is used to calculate the inverse Fourier transform of $X_{\Omega_{0}}(j \Omega)$ as follows

$$
x_{a}(t)=F^{-1}\left(X_{\Omega_{0}}(j \Omega)\right)=2 \pi x(t) \cdot \frac{1}{\Omega_{0}} \delta_{T}(t)=\operatorname{Tx}(t) \delta_{T}(t)=\sum_{n=-\infty}^{\infty} T x(n T) \delta(t-n T) .
$$

Consider $x(n)=T x(n T)$, then

$$
x(n)=\frac{1}{2 \pi} \int_{-\pi / T}^{\pi / T} X_{\Omega_{0}}(j \Omega) e^{j \Omega n T} d T \Omega=\frac{1}{2 \pi} \int_{-\pi}^{\pi} X(j \omega) e^{j n \omega} d \omega,
$$

where $\omega=\Omega T$ is the digital frequency, $X(j \omega)=X_{\Omega} 0(j \Omega)$. From the Eq.12, we have

$$
\begin{aligned}
& X(j \omega)=X_{\Omega_{0}}(j \Omega)=\int_{-\infty}^{\infty} x_{a}(t) e^{-j \Omega t} d t=\int_{-\infty}^{\infty}\left[\sum_{n=-\infty}^{\infty} T x(n T) \delta(t-n T)\right] e^{-j \Omega t} d t \\
& =\sum_{n=-\infty}^{\infty} T x(n T) \int_{-\infty}^{\infty} \delta(t-n T) e^{-j \Omega t} d t=\sum_{n=-\infty}^{\infty} T x(n T) e^{-j n T \Omega}=\sum_{n=-\infty}^{\infty} x(n) e^{-j n \omega} .
\end{aligned}
$$

Eq.14 and Eq.13 together form the positive and negative transform of DTFT, shown as Fig.1(c).

\section{Sampling both in Frequency Domain and Time Domain}

According to the derivations of DFS and DTFT, sampling $X(j \Omega)$ equally spaced in the frequency 
domain, the result is denoted by $\widetilde{X}(k)$, will lead to the periodic extension of $x(n)$ in the time domain which is recognizable as $\tilde{x}(n)$. Might as well set the length of a finite sequence $x(n)$ for $N$. Since $X(j \omega)$ is periodic in $\omega$ with period $2 \pi$, it follows that $\widetilde{X}(k)$ can also be obtained by sampling $X(j \omega)$ at $N$ equally spaced points in one period with a frequency spacing of $2 \pi / N$.

Through sampling $X(j \omega)$ with uniformly-space in frequency domain, Eq.14 can be rewritten as

$$
\tilde{X}(k)=\left.X(j \omega)\right|_{\omega=k \cdot \frac{2 \pi}{N}}=\sum_{n=-\infty}^{\infty} \tilde{x}(n) e^{-j \frac{2 \pi}{N} n k}, k=-\infty \sim+\infty .
$$

Since summing or integrating of a periodic signal can be conducted in a single cycle, it follows that

$$
\tilde{X}(k)=\sum_{n=0}^{N-1} \tilde{x}(n) e^{-j \frac{2 \pi}{N} n k}, k=-\infty \sim+\infty
$$

From Eq.16, only $N$ independent values, distributing in one cycle, can be obtained, i.e.

$$
X(k)=\sum_{n=0}^{N-1} \widetilde{x}(n) e^{-j \frac{2 \pi}{N} n k}, k=0, \cdots, N-1 .
$$

Do those operations below to Eq.17

$$
\sum_{k=0}^{N-1} X(k) e^{-j \frac{2 \pi}{N} l k}=\sum_{n=0}^{N-1}\left[\sum_{n=0}^{N-1} X(k) e^{-j \frac{2 \pi}{N} l k}\right] e^{-j \frac{2 \pi}{N} l k}=\sum_{n=0}^{N-1} x(n) e^{j \frac{2 \pi}{N} k(l-n)}
$$

and

$$
\sum_{k=0}^{N-1} e^{j \frac{2 \pi}{N} k(l-n)}=\left\{\begin{array}{ll}
N, & l-n=0, N, 2 N \cdots \\
0, & \text { else }
\end{array} .\right.
$$

Consequently, $x(n)$ and $\widetilde{x}(n)$ are related by

$$
\begin{aligned}
& x(n)=\sum_{k=0}^{N-1} X(k) e^{-j \frac{2 \pi}{N} n k}, n=0, \cdots, N-1 . \\
& \widetilde{x}(n)=\sum_{k=0}^{N-1} X(k) e^{-j \frac{2 \pi}{N} n k}, n=-\infty \sim+\infty
\end{aligned}
$$

Clearly, Eq.16 and Eq.21 are together the DFS pair, meanwhile Eq.17 and Eq.20 the DFT pair, respectively shown as Fig.1(d) and Fig.1(e).

Likewise, based on FS, DFS and DFT can also be derived through doing the equal interval sampling to the time-domain signal.

\section{Summary}

Based on the teaching difficulty of digital signal and processing course, this paper presents a teaching method. From FT, the process and results of sampling a time (frequency) domain signal in the frequency (time) domain are analysed, which helps the students to understand the relationship between the five Fourier transforms better. What we hope is to improve the teaching efficiency. 


\section{Acknowledgements}

This work was financially supported by the Education Teaching Reform Project of North China Electric Power University(Baoding)(NCEPU(baoding) university education file[2014] No. 1)

\section{References}

[1] Wang X G, Jin X Z and Bai K. Discussions about the Teaching Strategy of Fourier Transform: Advances in Social and Behavioral Sciences, Vol. 9 (2015), p.193-197.

[2] YANG Y L, LI J: Research on Teaching Methods for Digital Signal Processing Course(in Chinese), Journal of EEE, Vol.35, No. 4(2013), p. 72-74.

[3] LU D, LAN C F, YANG G X: Discussion on Large Class Teaching Model and Practice for Digital Signal Processing Course(in Chinese),China Electric Power Education, No. 16(2013), p. 86-87.

[4] Oppenheim A V, Schafer R W: Discrete-Time Signal Processing(3rd ed.)(Publishing House of Electronics Industry, Beijing, 2011 )

[5] HU G H : Introduction to digital signal processing(in Chinese)(Tsinghua University press, Beijing 2011). 